\title{
Oxygen abundances of zCOSMOS galaxies at $z \sim 1.4$ based on five lines and implications for the fundamental metallicity relation
}

\author{
Christian Maier ${ }^{1}$, Simon J. Lilly ${ }^{2}$, Bodo L. Ziegler ${ }^{1}$ and \\ zCOSMOS team \\ ${ }^{1}$ Department for Astrophysics, University of Vienna, \\ Türkenschanzstr. 17, 1180 Vienna, Austria \\ email: christian.maier@univie.ac.at \\ ${ }^{2}$ Institute of Astronomy, ETH Zurich, 8093 Zurich, Switzerland
}

\begin{abstract}
A relation between the stellar mass $M$ and the gas-phase metallicity $Z$ of galaxies, the MZR, is observed up to higher redshifts. It is a matter of debate, however, if the SFR is a second parameter in the MZR. To explore this issue at $z>1$, we used VLT-SINFONI near-infrared (NIR) spectroscopy of eight zCOSMOS galaxies at $1.3<z<1.4$ to measure the strengths of four emission lines: $\mathrm{H} \beta$, [OIII] $\lambda 5007, \mathrm{H} \alpha$, and [NII] $\lambda 6584$, additional to [OII] $\lambda 3727$ measured from VIMOS. We derive reliable $\mathrm{O} / \mathrm{H}$ metallicities based on five lines, and also SFRs from extinction corrected $\mathrm{H} \alpha$ measurements. We find that the MZR of these star-forming galaxies at $z \approx 1.4$ is lower than the local SDSS MZR by a factor of three to five, a larger change than reported in the literature using $[\mathrm{NII}] / \mathrm{H} \alpha$-based metallicities from individual and stacked spectra. Correcting N2-based O/Hs using recent results by Newman et al. (2014), also the larger FMOS sample at $z \sim 1.4$ of Zahid et al. (2014) shows a similar evolution of the MZR like the zCOSMOS objects. These observations seem also in agreement with a non-evolving FMR using the physically motivated formulation of the FMR from Lilly et al. (2013).
\end{abstract}

\section{Introduction}

In the local universe, the metallicity at a given mass also depends on the SFR of the galaxy (e.g., Mannucci et al. 2010), i.e., the SFR appears to be a "second-parameter" in the mass-metallicity relation (MZR). Mannucci et al. (2010) claimed that this local $Z(M, S F R)$ is also applicable to higher redshift galaxies, and coined the phrase fundamental metallicity relation (FMR) to denote this epoch-invariant $Z(M, S F R)$ relation. Lilly et al. (2013) showed that a $Z(M, S F R)$ relation is a natural outcome of a simple model of galaxies in which the SFR is regulated by the mass of gas present in a galaxy.

Most studies of the $\mathrm{Z}(\mathrm{M}, \mathrm{SFR})$ at $z \sim 1.4$ were based on small samples or samples with limited spectroscopic information, producing contradictory results. Except for the $z \sim 1.4$ study of Maier et al. (2006), the derived $\mathrm{O} / \mathrm{H}$ metallicities in the literature at $z \sim 1.4$ have been based just on $[\mathrm{NII}] / \mathrm{H} \alpha$, via the N2-method (Pettini \& Pagel 2004). However, Newman et al. (2014) found that the MZR at high $z$ determined using the N2-method might be up to a factor of three times too high in terms of metallicity, when the effects of both photoionization and shocks are not taken into consideration.

\section{Results}

We have carried out NIR follow-up spectroscopy with VLT-SINFONI of eight massive star-forming galaxies at $1.3<z<1.4$, selected from the zCOSMOS-bright sample (Lilly et al. 2009) based on their [OII] $\lambda 3727$ emission line observed with VIMOS. Observations 


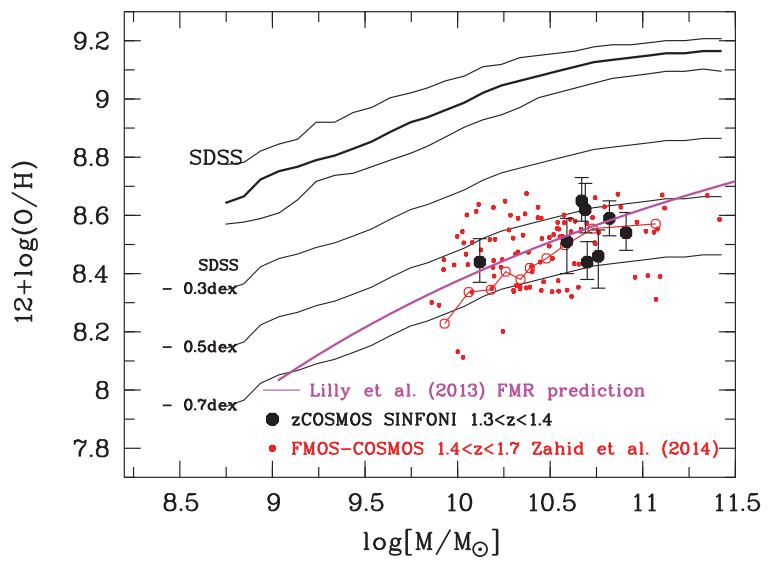

Figure 1. The median local MZR and $1 \sigma$ values of local SDSS galaxies from Tremonti et al. (2004) are shown by the three upper black lines, while the three thinner lower black lines show the median SDSS MZR shifted downward by $0.3,0.5$ and 0.7 dex, respectively. The Zahid et al. (2014) N2-based O/Hs are corrected for the N2-calibration issues discussed in Newman et al. (2014), and shown as red dots for the individual measurements, and as open red circles for the $\mathrm{O} / \mathrm{Hs}$ derived from the stacked spectra. The observed MZR at $z \approx 1.4$ is lower than the local SDSS MZR by a factor of three to five, and is also in agreement with the FMR prediction of Lilly et al. (2013).

with SINFONI in two bands ( $\mathrm{J}$ to observe the $\mathrm{H} \beta$ and $[\mathrm{OIII}] \lambda 5007$, and $\mathrm{H}$ to observe $\mathrm{H} \alpha$ and $[\mathrm{NII}] \lambda 6584)$ were performed between November 2013 and January 2014. SFRs, masses and $\mathrm{O} / \mathrm{H}$ metallicities were measured as described in Maier et al. (2014).

In Fig. 1 we compare the MZR of galaxies at $1.3<z<1.7$ with the relation of SDSS. Stellar masses are converted to or computed with a Salpeter (1955) IMF, O/Hs are computed or converted to the Kewley \& Dopita (2002) calibration. The Zahid et al. (2014) N2-based O/Hs were converted to the Kewley \& Dopita (2002) calibration and then systematically corrected to 0.4 dex lower metallicities, in agreement with recent results of Newman et al. (2014) who found that the MZR at $z>1$ determined using the N2-method might be $2-3$ times too high in terms of metallicity. We also overplot (as a magenta line) the Lilly et al. (2013) prediction of a non-evolving Z(M,SFR), the case represented by the dashed lines in Fig. 7 of Lilly et al. (2013). The $z \sim 1.4$ observational data shown in Fig. 1 are in agreement with this FMR prediction.

\section{References}

Kewley, L. J. \& Dopita, M. A. 2002, ApJS, 142, 35

Lilly, S. J., Le Brun, V., Maier, C., et al. 2009, ApJS, 184, 218

Lilly, S. J., Carollo, C. M., Pipino, A., et al. 2013, ApJ, 772, 119

Maier, C., Lilly, S. J., Carollo, C. M., et al. 2006, ApJ, 639, 858

Maier, C., Lilly, S. J, Ziegler, B. L., et al. 2014, ApJ, 792, 3

Mannucci, F., Cresci, G., Maiolino, R., et al. 2010, MNRAS, 408, 2115

Newman, S. F., Buschkamp, P., Genzel, R., et al. 2014, ApJ, 781, 21

Pettini, P. \& Pagel, B. E. J.. 2004, MNRAS, 348, 59

Salpeter, E. E. 1955, ApJ, 121, 161

Tremonti, C. A., Heckman, T. M., Kauffmann, G., et al. 2004, ApJ, 613, 898

Zahid, H. J., Kashino, D., Silverman, J. D., et al. 2014, ApJ, 792, 75 